\title{
Distillation and survival of strange-quark-matter droplets in ultrarelativistic heavy-ion collisions
}

\author{
Carsten Greiner \\ Institut für Theoretische Physik, Universität Erlangen, Staudtstrasse 7, D-8520 Erlangen, Germany \\ Horst Stöcker \\ Institut für Theoretische Physik, J. W. Goethe-Universität, Robert-Mayer-Strasse 10, \\ D-6000 Frankfurt am Main 11, Germany \\ (Received 26 February 1991)
}

\begin{abstract}
Recently it has been suggested that rather cold droplets of absolutely stable or metastable strangequark matter may be distilled in heavy-ion collisions during the phase transition from a baryon-rich quark-gluon plasma (QGP) to hadron matter. Here we present a model describing the hadronization of the QGP through particle emission, which is based solely on thermodynamical arguments. Pions and $K^{+}$'s and $K^{0}$ 's carry away entropy and antistrangeness from the system, thus facilitating the cooling process and the strangelet formation. Our results are supported by revised more sophisticated rate calculations. Two rather unexpected results are obtained when this model is applied to the investigation of strangelet production. The strangeness separation mechanism and the formation process works well even for higher initial entropies per baryon, tantamount to higher bombarding energies. The surviving strangelets have a rather high strangeness content, $f_{s} \sim 1.2-2$ [i.e., $\left.Z / A \sim(-0.1)-(-0.5)\right]$. Hence droplets of strange-quark matter with a baryon number of $\sim 10-30$ and with a negative charge may be produced. They may serve as a unique signature for the transient formation of a quark-gluon plasma in heavy-ion collisions.
\end{abstract}

\section{INTRODUCTION AND MOTIVATION}

One important goal in ultrarelativistic heavy-ion physics is the observation of a temporarily created quarkgluon plasma (QGP) [1]. An enhanced production of strange particles from the QGP, especially an enhanced $K^{+} / \pi^{+}$ratio [2], and the $J / \psi$ suppression [3] were proposed as possible signatures for such a novel state of deconfined, strongly interacting matter. The observed $J / \psi$ suppression [4] and the observed $K^{+} / \pi^{+}$ratio [5] had first raised premature euphoria that such a state has indeed been unambiguously observed. However, it was subsequently shown that these data-and also most of the other proposed signals for QGP creation-can be understood as being due to the formation of a very (energy-) dense and hot region of matter consisting of confined hadrons [1].

Therefore, it seems that perhaps the only unambiguous way to detect the transient existence of a QGP might be the experimental observation of remnants ("ashes" of the QGP, [1]), such as the formation of strange-quark-matter droplets: It has been proposed that strange-quark-matter droplets ("strangelets") at zero temperature and in $\beta$ equilibrium might be absolutely stable $[6,7]$. Such a scenario would be of fundamental importance: It would for example explain a large fraction of the nonobservable mass of the Universe in certain cosmological models, and would modify substantially our understanding of the structure and stability of neutron stars. We have recently argued [8,9] that such strangelets might play an important role in ultrarelativistic heavy-ion collisions. Here one expects a total net strangeness of zero, a high temper- ature and no $\beta$-chemical equilibrium. How can these speculative objects then be produced in such experiments?

Let us briefly summarize what a stable or metastable strangelet might look like: Think of bulk objects, containing a large number of quarks $(u \ldots u, d \ldots d, s \ldots s)$, so-called multiquark droplets. Multiquark states consisting only of $u$ and $d$ quarks must have a mass larger than ordinary nuclei, otherwise normal nuclei would be unstable. However, the situation is different for droplets of strange-quark matter, which would contain approximately the same amount of $u, d$, and $s$ quarks. These exotic states have not yet been observed, although QCD allows for their existence - the smallest of these objects would be the $H$ dibaryon proposed by Jaffe [10].

There are two reasons why such states could be relatively stable.

(1) The decay of an $s$ quark into a $d$ quark would be suppressed or forbidden because the lowest single-particle states are occupied.

(2) The strange quark mass is lower than the Fermi energy of the $u$ or $d$ quark in such a quark droplet; the opening of a new flavor degree of freedom tends to lower the Fermi energy and hence also the mass of the strangelet.

If the mass of a strangelet is smaller than the mass of the corresponding ordinary nucleus with the same baryon number, the strangelet would be absolutely stable and thus be the true ground state of nuclear matter [6]. Presently such a scenario cannot be ruled out. Normal nuclei could only transform into these novel states by a collective weak decay; the decay into one single $\Lambda$ parti- 
cle would be energetically forbidden. The time scale for such collective decays is extremely large; hence, normal nuclei would remain intact.

On the other hand, it is also conceivable that the mass per baryon of a strange droplet is lower than the mass of the strange $\Lambda$ baryon, but larger than the nucleon mass. The droplet is then in a metastable state, it cannot decay into $\Lambda$ 's [11].

If popular models of hadrons are extrapolated to strange-quark droplets, such as, e.g., the MIT bag model, both of the above considered pictures turn out to be possible. However, metastable strangelets relax somewhat the stringent conditions on the choice of parameters of the bag model required by an absolutely stable state [7], namely small bag constants $B^{1 / 4} \lesssim 150 \mathrm{MeV}$, which would give very small transition temperatures to a QGP, $T_{c} \sim 100 \mathrm{MeV}$.

There are two related arguments why strangelets could be produced in ultrarelativistic heavy-ion collisions, although here the initial conditions are such that there is no strangeness in the system at all, and that even after the collision, there is no net strangeness: Equal amounts of strange and antistrange quarks are abundantly produced in a hot QGP [2]. The strangeness saturates the phase space in a baryon-rich environment after a very short equilibrium time, $t \simeq 10^{-23} \mathrm{sec}$, which may actually be shorter than the duration of a nuclear collision. It has been demonstrated [8] that the phase transition from such a QGP to hadron matter will yield a large antistrangeness content in the hadron phase while the quarkgluon plasma retains a large net strangeness excess. This distillation happens even if the total net strangeness of the combined QGP and hadron fluid is equal to zero. The separation process takes place, if the plasma phase (when the combined system carries a net positive baryon number) is energetically favored for the $s$ quark as compared to the confined phase where the $s$ quarks reside in antikaons $\left(K^{-}\right)$and heavy hyperons. The distillation mechanism can be viewed as being due to the associated production of kaons (containing $\bar{s}$ quarks) in the hadron phase with the $s$ quarks remaining in the QGP droplets, which therefore develop a net strangeness surplus.

Furthermore, rapid kaon emission leads to a finite net strangeness of the expanding (combined hadron plus quark) system $[9,12]$. This, in turn, results in an even stronger enhancement of the $s$-quark abundance in the quark phase. This prompt kaon (and, of course, also pion) emission may cool the quark phase, which then condenses into metastable or stable droplets of strangequark matter [9].

Recently Barz et al. [13] presented rate equation calculations, which suggested that nucleon evaporation heats up nonstrange quark droplets, which in response evaporate completely. This analysis applied to evaporation of nucleons only. They concluded that the formation of a long-lived cold plasma droplet is, even for nonzero net strangeness, very unlikely. However, this was demonstrated in Ref. [13] only for large bag constants $\left(B^{1 / 4}=235 \mathrm{MeV}\right)$. It confirms our earlier results [9], namely that then strangelets would in fact neither be stable nor metastable: The mass of a quark-matter drop- let (at zero temperature) is much too large to be stable, $E / A \sim 6 B^{1 / 4} \sim 1400 \mathrm{MeV}$, as compared to the nucleon mass, $940 \mathrm{MeV}$. The surplus of $\sim 450 \mathrm{MeV}$ nucleon can heat up the cold blob quickly.

On the other hand, explicit cooling calculations have been performed for a small bag constant $\left(B^{1 / 4}=145\right.$ $\mathrm{MeV})$ [14] for rather cold $(T \sim 20-50 \mathrm{MeV})$ and very large $\left(A \sim 10^{50}\right)$ strange-quark-matter globs formed in the early Universe. An effective nucleon binding energy $I_{n}=m_{n}-3 \mu_{q}>0$ is found due to detailed balance for nucleons and antikaons evaporated from the surface.

In the present article we demonstrate that a careful treatment of the strangeness evolution incorporates both results. A nonstrange quark-gluon-plasma droplet formed in relativistic heavy-ion collisions can, even at very high entropies, evolve into a blob of strange-quark matter with negative charge.

We will show below (see Fig. 8) that the chemical potentials $\mu_{q}$ and $\mu_{s}$ of the evolving droplets (as the time is increasing, i.e., $t \rightarrow \rightarrow^{\prime \prime} \infty$ ", and the temperature drops to $T \sim 10-20 \mathrm{MeV}$ ) are

$$
\begin{array}{ll}
\text { (1) } \mu_{q}(t \rightarrow \infty) \sim 200-275 \mathrm{MeV}, \\
\mu_{s}(t \rightarrow \infty) \sim 300-325 \mathrm{MeV} \quad\left(B^{1 / 4}=145 \mathrm{MeV}\right) ; \\
\text { (2) } \mu_{q}(t \rightarrow \infty) \sim 200-250 \mathrm{MeV}, \\
\mu_{s}(t \rightarrow \infty) \sim 350-400 \mathrm{MeV} \quad\left(B^{1 / 4}=160 \mathrm{MeV}\right)
\end{array}
$$

In these cases $I_{n} \sim 120-350 \mathrm{MeV}$ is indeed positive at the late stages of the time evolution. Thus, even if the initial baryon emission is accompanied by a heating of the plasma, the long-time behavior can be quite different.

To illustrate how stable strangelets cool and survive, let us consider for the moment just nucleon evaporation: The energy per baryon of a strangelet at small temperature and zero pressure is approximated by $[6,7]$.

$$
\frac{E}{A}=\left(\frac{E}{A}\right)_{T=0}+\gamma T^{2}, \quad \gamma \sim \frac{1}{20} \frac{1}{\mathrm{MeV}} .
$$

The emission of a nucleon will decrease the energy per baryon of the blob:

$$
\Delta\left(\frac{E}{A}\right)=\frac{I_{n}}{A(T)} \Delta A, \Delta A<0 .
$$

In addition, the differential of Eq. (1) is $\Delta(E / A)=2 \gamma T \Delta T$. Therefore $\Delta A<0$ yields $\Delta T<0$ for $I_{n}>0$. The decrease of the baryon number as a function of temperature, $A(T)$, and the effective binding energy, $I_{n}$ can be used to calculate the asymptotic baryon number $A^{\text {final }}$ in the droplet $(T \rightarrow 0$, i.e., $t \rightarrow \infty)$. If $I_{n}$ is assumed to stay constant (actually $\mu_{q}$ will decrease and hence $I_{n}$ will increase even further), the integration from the initial temperature $T_{i}$ to $T=0$ can be performed analytically. This yields the final baryon number of the strangelet:

$$
A^{\mathrm{final}}=A\left(T_{i}\right) \exp \left[\frac{-\gamma T_{i}^{2}}{I_{n}}\right] .
$$


For $I_{n} \sim 250 \mathrm{MeV}$ and $T_{i} \sim 20 \mathrm{MeV}$, a total of $\sim 92 \%$ of the initial baryon number would remain in the droplet. Hence, evaporation of baryons from quark matter will be suppressed at very low temperatures, because there simply is not enough energy available to power the evaporation [6].

Further cooling of the strangelets will be achieved by pion, photon, and dilepton evaporation. These points were left out in the analysis of Madsen et al. [14]. The pion mass $\sim 138 \mathrm{MeV}$ is smaller than the effective binding energy $I_{n}$ of the nucleons, hence the suppression of the pion evaporation $\sim e^{-m_{\pi} / T}$ is not as effective. Photons and dileptons can be emitted from the interior of the blob-their production is not restricted to the surface region, because their mean free paths are much larger $(\lambda \sim 50-100 \mathrm{fm})$ than the size of systems created in heavy-ion collisions $(R<10 \mathrm{fm})$.

Let us now study in more detail the distillation and possible survival of strangelets. One of the main results of the rate calculations $[2,15]$ is that particle yields calculated from the nonequilibrium evaporation off the plasma agree very well with the equilibrium yields obtained for the hadron gas.

\section{THE MODEL}

The hadronization transition of a QGP droplet formed in heavy-ion collisions has often been described by simple geometric and statistical equilibrium models. The participant fireball is assumed to be in thermodynamic equilibrium. A hydrodynamic expansion is often assumed, which is substantially modified by the phase transition from QGP to the hadronic phase [16,17]. A more realistic scenario must take into account the competition of the collective expansion with the particle radiation from the surface of the hadronic fireball before "freeze-out." A simple "cascade" model was presented [18], where the emission and reabsorbtion of pions from the surface of the hot plasma was studied. However, for the present purpose, evaporation of other hadrons must also be taken into account. Nucleons and strange particles (e.g., kaons and hyperons) are particularly important. One important outcome of the cascade model [18] was the observation that the total entropy is approximately constant during the hadronization. Chemical nonequilibrium model calculations $[2,15,17]$ suggested furthermore that chemical equilibrium is reached in the hadron phase, if the system evolves from the deconfined phase: gluon fusion yields fast equilibration of strangeness. The subsequent rehadronization affects that the strange hadron yields closely approach the hadron equilibrium yields from above. Hence, a two-phase equilibrium description gives results which agree with nonequilibrium models.

However, such a model must incorporate the evaporation of hadrons from the total system. We will show that it does indeed reproduce all features of the rate equation models. The nonequilibrium evaporation is incorporated by rapid freeze-out of hadrons from the hadron phase surrounding the QGP droplet at the Gibbs phase transition point. This scenario is visualized in Fig. 1 .

The global properties of the two-phase system then change in time in accord with the following differential equations for the baryon number, the entropy, and the net strangeness number of the total system:

$$
\begin{aligned}
& \frac{d}{d t} A^{\text {tot }}=-\Gamma A^{\text {had }}, \\
& \frac{d}{d t} S^{\text {tot }}=-\Gamma S^{\text {had }}, \\
& \frac{d}{d t}\left(N_{s}-N_{\bar{s}}\right)^{\text {tot }}=-\Gamma\left(N_{s}-N_{\bar{s}}\right)^{\text {had }},
\end{aligned}
$$

where $\Gamma=\left(1 / A^{\text {had }}\right)\left(\Delta A^{\text {had }} / \Delta t\right)_{\mathrm{ev}}$ is the effective ("universal") rate (of converted hadron gas volume) of particle evaporated from the hadron phase.

These three equations may easily be combined in a physically illustrative form:

$\frac{d}{d t}\left[\frac{S}{A}\right]^{\text {tot }}=-\Gamma \lambda^{\text {had }}\left(1-\lambda^{\text {had }}\right)\left[\left(\frac{S}{A}\right]^{\text {had }}-\left(\frac{S}{A}\right)^{\mathrm{QGP}}\right]$,

$\frac{d}{d t} f_{s}^{\mathrm{tot}}=-\Gamma \lambda^{\mathrm{had}}\left(1-\lambda^{\mathrm{had}}\right)\left(f_{s}^{\mathrm{had}}-f_{s}^{\mathrm{QGP}}\right)$.

Here $\lambda^{\text {had }}=1-\lambda^{\mathrm{QGP}}=A^{\text {had }} / A^{\text {tot }}$ is the ratio of baryon number contained in the hadron phase over the total baryon number in the system, $f_{s}$ defines the total net strangeness content of the system, i.e., $f_{s}=\left(N_{s}-N_{\bar{s}}\right) / A^{\text {tot }}$, and $S / A$ is the entropy per baryon. What is the physical content of Eq. (6)? Obviously, $\Gamma$, $\lambda^{\text {had }}$, and $\left(1-\lambda^{\text {had }}\right)$ are larger than zero. Therefore the total entropy of the remaining system $(S / A)^{\text {tot }}$ will decrease $\left[(d / d t)(S / A)^{\text {tot }}<0\right]$; i.e., the system will be cooled, if and only if the specific entropy per baryon in the hadron phase exceeds that in the quark phase, $(S / A)^{\text {had }}>(S / A)^{\mathrm{QGP}}$. The system will reheat, i.e, $(S / A)^{\text {tot }}$ will increase $\left[(d / d t)(S / A)^{\text {tot }}>0\right]$, if and only if $(S / A)^{\text {had }}<(S / A)^{\mathrm{QGP}}$.

Figure 2(b) shows the ratio $R=(S / A)^{\mathrm{QGP}} /(S / A)^{\text {had }}$ versus the net strangeness fraction $f_{s}^{\mathrm{QGP}}$ in the plasma droplet for various temperatures along the Gibbs phasetransition surface in the baryochemical potentials $\mu_{q}$ and $\mu_{s}$ for a bag constant $B^{1 / 4}=180 \mathrm{MeV}\left(=136 \mathrm{MeV} \mathrm{fm}^{-3}\right)$. Note that $R>1$ or $R<1$ corresponds to reheating or cooling, respectively. For high temperatures the system

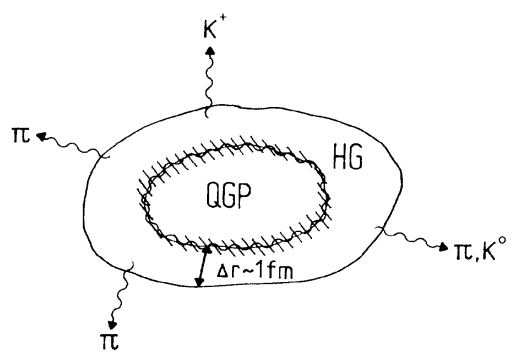

FIG. 1. Hadron fluid surrounds the QGP at the phase transition. Particles evaporate from the hadronic region. New hadrons emerge out of the plasma by hadronization. 


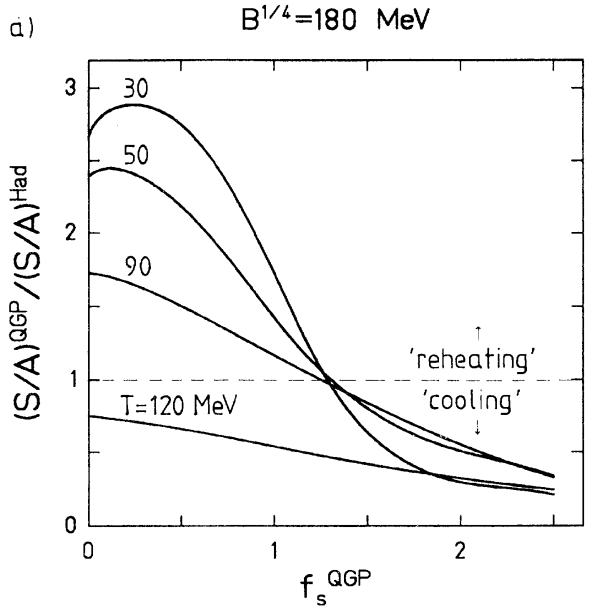

b)

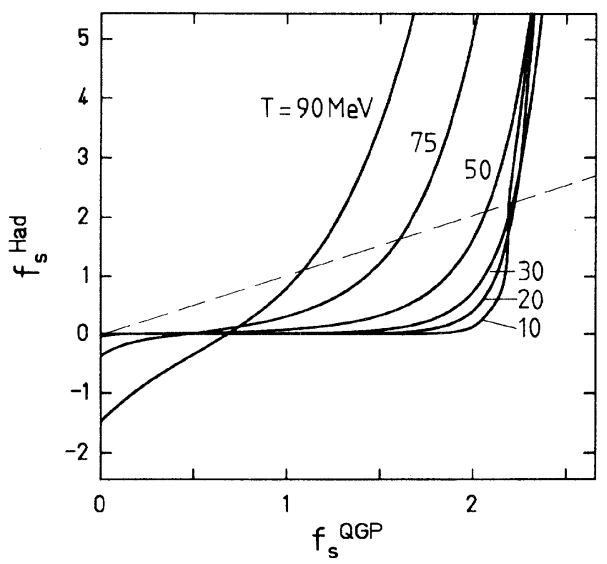

FIG. 2. (a) The ratio of the entropy per baryon of the two phases versus $f_{s}^{\text {QGP }}$ is shown along the Gibbs phase coexistence region, i.e, for different temperatures. A bag constant of $B^{1 / 4}=180 \mathrm{MeV}$ is employed for the sake of illustration. (b) The dependence of $f_{s}^{\text {had }}$ versus $f_{s}^{\text {QGP }}$ is shown along the phase coexistence surface $\left(B^{1 / 4}=145 \mathrm{MeV}\right)$, i.e, for different temperatures. In the interesting regime for heavy-ion collisions, note that $f_{s}^{\text {QGP }}>f_{s}^{\text {had }}$.

always cools; for lower temperatures cooling is observed only for $f_{s}^{\text {QGP }} \gtrsim 1.3$ at this bag constant. For the small bag constant, i.e. for absolutely stable strangelets, the system always cools.

A similar argument holds for the net strangeness fraction $f_{s}^{\text {tot }}$ of the system, Eq. (7). $f_{s}^{\text {tot }}$ will always increase or decrease, respectively, with time, if the fraction $f_{s}^{\text {had }}$ is smaller or larger, respectively, than $f_{s}^{\text {QGP }}$. We have shown in Ref. [8] that kaons $\left(K^{+}, K^{0}\right)$ are abundantly created in the hadron phase. Their presences enforces $f_{s}^{\text {had }}<0$ early on. Also later on $f_{s}^{\text {had }}$ remains smaller than $f_{s}^{\mathrm{QGP}}$, and therefore, the first scenario holds: $f_{s}^{\text {tot }}$ increases as a function of time $\left[(d / d t) f_{s}^{\text {tot }}>0\right]$.

Figure 2(b) shows the dependence of $f_{s}^{\text {had }}$ versus $f_{s}^{\text {QGP }}$ along the phase coexistence surface for different temperatures. The strangeness fraction $f_{s}^{\text {had }}$ in the hadron phase is negative $\left(f_{s}^{\text {had }}=-1.4\right)$ for $f_{s}^{\mathrm{QGP}}=0$ at $T \sim 100 \mathrm{MeV}$. Hence, the separation mechanism of the strange and antistrange quarks [8] at higher temperatures can be read off at once from Fig. 2(b). A strong increase in the strangeness content of the plasma is predicted: For all temperatures $T<50 \mathrm{MeV}$ and plasma fractions $f_{s}^{\mathrm{QGP}}<2$ the net strangeness content of the plasma $f_{s}^{\text {QGP }}$ is greater than $f_{s}^{\text {had }}$.

The three equations (3)-(5) [or (3), (6), and (7)] constitute a set of coupled differential equations for $A^{\text {tot }}$, $(S / A)^{\text {tot }}$ and $f_{s}^{\text {tot }}$. The latter two are given in terms of the hadron and quark phase content by

$$
\begin{aligned}
& \left(\frac{S}{A}\right)^{\text {tot }}=\left(\frac{S}{A}\right)^{\mathrm{QGP}}\left(\mu_{q}, \mu_{s}, T\right)\left(1-\lambda^{\text {had }}\right) \\
& +\left(\frac{S}{A}\right)^{\text {had }}\left(\mu_{q}, \mu_{s}, T\right) \lambda^{\text {had }}, \\
& f_{s}^{\text {tot }}=f_{s}^{\mathrm{QGP}}\left(\mu_{q}, \mu_{s}, T\right)\left(1-\lambda^{\text {had }}\right) \\
& +f_{s}^{\text {had }}\left(\mu_{q}, \mu_{s}, T\right) \lambda^{\text {had }} .
\end{aligned}
$$

Although the baryon number $A$ and the strangeness $f_{s}$ are conserved under strong interactions (and, as motivated above, also the total amount of entropy $S / A$ throughout the expansion), their value in the system changes with time according to Eqs. (3), (6), and (7) due to the evaporation process. This calculation requires solving simultaneously the equations of motion and the Gibbs phase equilibrium conditions, which specify the intrinsic variables of the functions $(S / A)^{\mathrm{QGP} / \mathrm{had}}\left(\mu_{q}, \mu_{s}, T\right)$ and $f_{s}^{\mathrm{QGP} / \mathrm{had}}\left(\mu_{q}, \mu_{s}, T\right)$.

When the phase coexistence region is reached in the hydrodynamic expansion of the plasma droplet, baryon number and entropy conservation force the system along the phase boundary by converting plasma into hadron gas. The Gibbs conditions read

$$
\begin{aligned}
& P^{\mathrm{QGP}}\left(\mu_{q}, \mu_{s}, T\right)=P^{\mathrm{had}}\left(\mu_{q}, \mu_{s}, T\right), \\
& T^{\mathrm{QGP}}=T^{\mathrm{had}}, \\
& \mu_{q}^{\mathrm{QGP}}=\mu_{q}^{\mathrm{had}}, \\
& \mu_{s}^{\mathrm{QGP}}=\mu_{s}^{\text {had }} .
\end{aligned}
$$

For a given entropy and baryon number, the temperature $T$ and the baryochemical potential $\mu_{q}$ specify the volume occupied by the two phases. The strange chemical potential $\mu_{s}$ needs to be specified to ensure the strangeness conservation.

The equation of state used consists of the bag model for the QGP and a mixture of relativistic Bose-Einstein and Fermi-Dirac gases of the well-established nonstrange and strange hadrons up to $M \sim 2 \mathrm{GeV}$ for the hadron matter [9]. The thermodynamic functions for the hadron gas are used in Hagedorn's eigenvolume corrections to take into account interactions between the hadrons [19].

The above equations (3), (6), and (7) which govern the evolution of the system from the initial, hot plasma droplet to the final state, be it a completely evaporated hadron gas or a remaining cold strangelet, can be discretized in finite-time steps $\Delta t$ for the numerical integration: 


$$
\begin{aligned}
& A^{\text {tot }}(t+\Delta t)=A^{\text {tot }}(t)\left(1-\lambda^{\text {had }} \Gamma \Delta t\right), \\
& {\left[\frac{S}{A}\right]^{\text {tot }}(t+\Delta t)=\left[\left[\frac{S}{A}\right]^{\text {tot }}(t)-\left[\frac{S}{A}\right]^{\text {had }}(t) \lambda^{\text {had }} \Gamma \Delta t\right] /\left(1-\lambda^{\text {had }} \Gamma \Delta t\right),} \\
& f_{s}^{\text {tot }}(t+\Delta t)=\left[f_{s}^{\text {tot }}(t)-f_{s}^{\text {had }}(t) \lambda^{\text {had }} \Gamma \Delta t\right] /\left(1-\lambda^{\text {had }} \Gamma \Delta t\right),
\end{aligned}
$$

The plasma is initialized with the values $A\left(t_{0}\right)$, $\left(S^{\text {tot }} / A\right)\left(t_{0}\right)$ and $f_{s}^{\text {tot }}\left(t_{0}\right)$ at a time $t_{0} \sim 5 \mathrm{fm} / c$ [Eq. (8) with $\left.\lambda_{B}^{\mathrm{QGP}}=1\right]$.

Now the baryon fraction $\lambda_{\text {had }}$ and the rate $\Gamma$ need to be specified. The system then expands spherically until the hadron volume has reached a thickness of $\Delta r \sim 1 \mathrm{fm}$ (this is an ad hoc assumption; however, the results depend only weakly on $\Delta r$ ). Then

$$
\begin{aligned}
\lambda_{B}^{\text {had }}= & \frac{4}{3}\left[\left(R^{\mathrm{QGP}}+\Delta r\right)^{3}-\left(R^{\mathrm{QGP}}\right)^{3}\right] \\
& \times \rho_{B}^{\mathrm{had}}\left(\mu_{q}, \mu_{s}, T\right) / A^{\mathrm{tot}}(t),
\end{aligned}
$$

where the radius $R^{\mathrm{QGP}}=R^{\mathrm{QGP}}(t)$ of the plasma drop is given by

$$
\begin{aligned}
R^{\mathrm{QGP}}(t)= & {\left[\frac{3}{4 \pi} \frac{1}{\rho_{B}^{\mathrm{QGP}}\left(\mu_{q}(t), \mu_{s}(t), T(t)\right)}\right.} \\
& \times \lambda^{\left.\mathrm{QGP}(t) A^{\mathrm{tot}}(t)\right)^{1 / 3} .}
\end{aligned}
$$

A fraction $\Gamma \Delta t$ of the hadron gas is evaporated during each small step $\Delta t$, and the size of the hadronic volume changes according to Eq. (3) from $\Delta r$ to $\Delta r^{\prime}=\Delta r(1-\Gamma \Delta t)$. Simultaneously, the variables $T, \mu_{q}$, and $\mu_{s}$ [see Eq. (8)] change in the subsequent infinitesimal expansion. An average rate is used for all particles for the temperature considered at this time interval

$$
\Delta t \sim \frac{\Delta r-\Delta r^{\prime}}{\langle v\rangle} \sim\left[\frac{\langle m\rangle}{3 T}\right]^{1 / 2}\left(\Delta r-\Delta r^{\prime}\right),
$$

where $v$ and $m$ give the average velocity and mass of the hadrons evaporated from the surface. Note that this ansatz simply scales the time dependence of the global quantities and has no influence on their asymptotic behavior. However, unlike in normal hydrodynamical models, which neglect particle freeze-out during the expansion, here the volume increase competes with the decrease due to the freeze-out. Hence, the iteration of Eq. (8) and Eqs. (3), (6), and (7) yields the hadronization of the QGP and the particle emission off the hadron phase.

In this model the global-expansion ansatz dictates the time scale for the evaporation of the hadronic particles out of the hadron phase. Therefore the rates of the emitted particles depend on the chosen ansatz for the hydrodynamic expansion. A longitudinal expansion, as assumed in the Bjorken scenario [20], would drastically change the time dependence $(V \sim T)$. However, the final (total) yields are not effected by the ansatz.

Of course, this complicated self-consistent calculation can be approximated by the analogue of Eq. (2), with almost "asymptotic" chemical potentials once the high ini- tial temperature of the system drops to low temperatures, $T \leq 30 \mathrm{MeV}$ as $t \gtrsim 100 \mathrm{fm} / c=3 \times 10^{-22} \mathrm{sec}$. The integration from $t \sim 100 \mathrm{fm} / c$ to $t \rightarrow \infty$ can then be performed rather analytically. This yields the final baryon number of the remaining strangelet and other observables of interest.

\section{RESULTS}

As a first application of our model we study the hadronization of a hot, baryon-rich QGP with an initial baryon content of $A^{\text {tot }}\left(t_{0}\right)=36$, an initial entropy per baryon $(S / A)^{\text {tot }}\left(t_{0}\right)=25$, no initial net strangeness, $f_{s}^{\text {tot }}\left(t_{0}\right)=0$, for the case of a large bag constant of $B^{1 / 4}=235 \mathrm{MeV}$ where we have predicted total hadronization $[8,9,21]$ (these initial values are identical with those in Ref. [13] which support our earlier results). Figure 3(a) depicts the time evolution of the baryon number $A$ QGP of the quark droplet, its net strangeness content, $f_{s}^{\mathrm{QGP}}$, its radius, $R^{\mathrm{QGP}}$, and the temperature $T$. Indeed, the system hadronizes completely in $t \sim 5 \mathrm{fm} / c$.

The temperature of the system increases slightly, although one might have expected some cooling by the emission of particles. This reheating, however, is well known [16,13] for baryon-rich matter at large bag constants; it is due to the conservation of the large entropy content of the plasma. Rigorously, whether reheating or cooling does occur depends only on whether $(S / A)^{\mathrm{QGP}}$ is larger or smaller than $(S / A)^{\text {had }}$ at the phase transition point [see Eq. (6), Fig. 2(a) and the discussion above). It was pointed out in Ref. [9] that the strangelet formation goes hand in hand with strong cooling rather than reheating when the bag constant $B^{1 / 4}$ is small.

A strong increase of the net strangeness of the system is found confirming our previous work $[8,9]$. The plasma droplet acquires a strangeness fraction of $f_{s} \sim 1$ when the volume becomes small. This means that $f_{s}^{\text {QGP }}>f_{s}^{\text {had }}$ also for the large bag constant. The particle yields of the most frequently emitted species are shown in Fig. 3(b). Their time dependence is more or less as expected: the pions are by far the most quickly escaping and abundant particles followed by the nucleons, kaons, antikaons, and the hyperons (the particle decay of the resonances, e.g., the $\Delta$ 's, is taken into account in accord with the known decay modes).

These findings are in good agreement with the results of the rate equation models [15]. The only discrepancy is a factor of 2 in the kaon number [13,15]. Our $K^{+} / \pi^{+}$ ratio is found to be in the range $0.2-0.3$. It has recently been discovered [22] that this discrepancy was due to a trivial mistake in their calculation.

In conclusion, we have demonstrated that our model is in quantitative agreement with nonequilibrium models 
and describes the emission of hadronic particles based on thermodynamical arguments. In the following the model is applied to the search for the optimum strategy for strangelet formation, survival, and decay.

\section{STRANGELET CONDENSATION}

For the following arguments it is important to study the expansion of an initially hot QGP fireball with special attention to the evolution of the strangeness during the phase transition. Early nonequilibrium particle radiation off the hot, dense (baryon-rich) QGP fireball is expected to be important in the initial phase of the reaction $[9,12]$. Pions and kaons (containing an $\bar{s}$ quark) (and only a minor number of antikaons) are emitted from the surface of the plasma. Thus the QGP is enriched with finite net strangeness even before the phase transition point is reached. More kaons $(q \bar{s})$ than antikaons $(\bar{q} s)$ are emitted because the $\bar{K}$ 's containing a $\bar{q}$ quark are expected to be suppressed in baryon-rich matter (large $\mu_{q}$ ) by a factor

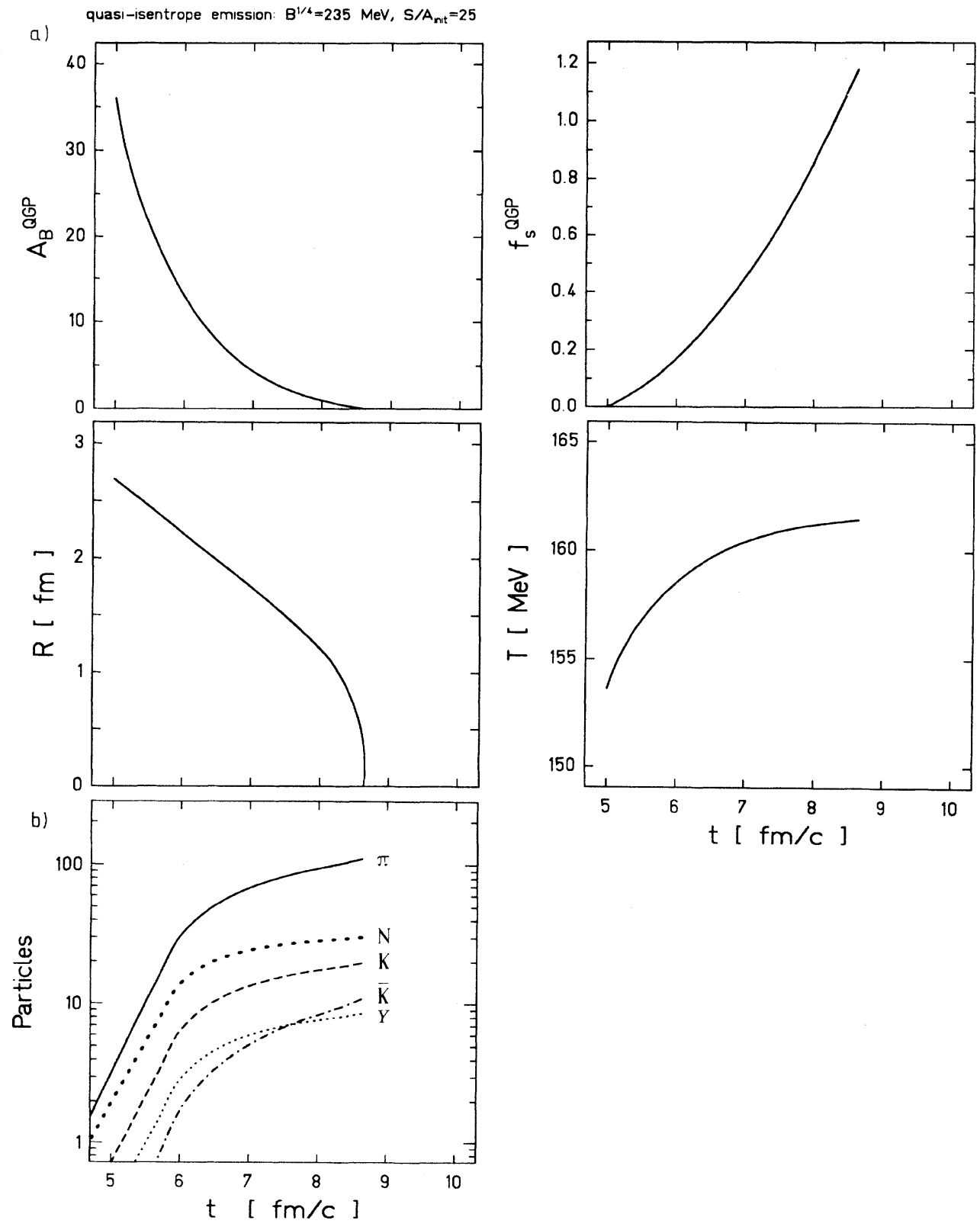

FIG. 3. (a) Baryon number, strangeness content, radius, and temperature of the quark glob during hadronization as a function of time. The initial baryon content is $A_{B}=36$, no initial net strangeness $\left[f_{s}^{\mathrm{QGP}}\left(t_{0}\right)=0\right]$ and the bag constant is $B^{1 / 4}=235 \mathrm{MeV}$. Note the strong increase of the strangeness content with time. (b) The evaporated particle number of the most abundant hadron species are shown. 
$\sim e^{-2 \mu_{q} / T}$ as compared to the $K$ 's. The net strangeness enrichment resulting from the early black-body radiation off the pure QGP droplet [23] has been estimated to be in the range $f_{s} \lesssim 0.5[9,12]$. These values have been used in the present model as input for the initial condition $f_{s}^{\mathrm{QGP}}\left(t_{0}\right)$.

Particle evaporation during the phase coexistence seems to be even more important: The system spends most of the expansion time in the two-phase coexistence due to entropy conservation [17]: Again, meson evapora-
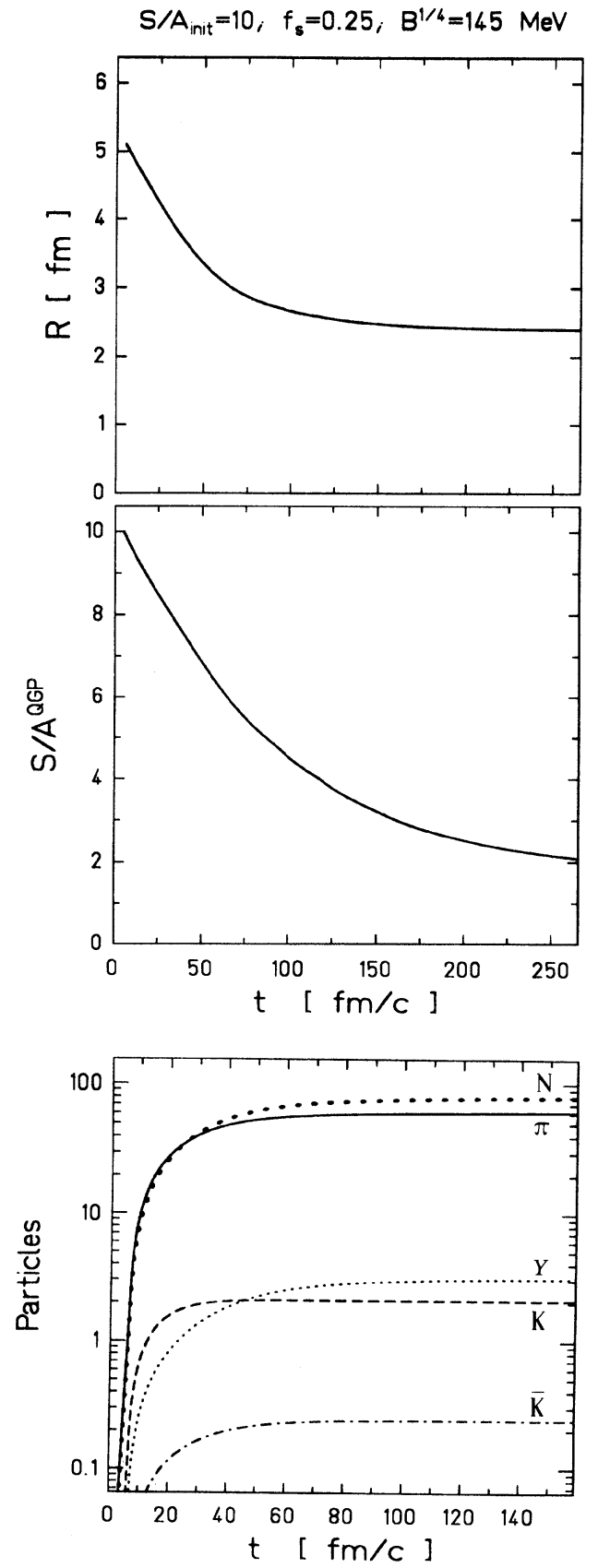

tion carries away entropy, energy, and antistrangeness. Hence the residual expanding fireball, which is in the mixed phase, loses entropy and charges up with net strangeness. It is shown below that this behavior further enhances the strangeness content in the quark phase to even higher values, i.e., to $f_{s}>1$, considerably larger than the $f_{s} \sim 0.7-0.9$ calculated in the simplified approach of Refs. [8,9]. Furthermore, the specific entropy in the quark phase drops strongly, while the remaining entropy flows into the hadron phase. The hadronization can thus

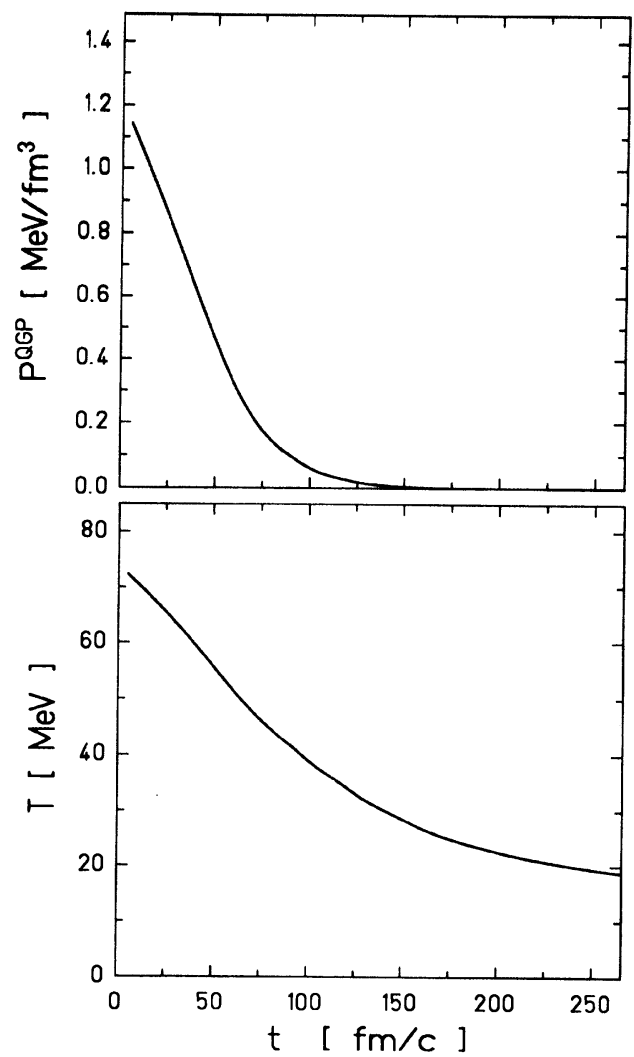

FIG. 4. The time evolution of several quantities $(R, P, S / A, T)$ are depicted for the case when a strangelet is distilled. The initial values are $A_{B}\left(t_{0}\right)=100,(S / A)\left(t_{0}\right)=10, f_{s}\left(t_{0}\right)=0.25\left(B^{1 / 4}=145 \mathrm{MeV}\right)$. The number of emitted particles are also shown versus time. 
result in the distillation of a rather cold strange-quarkmatter droplet. This debris of the quark-gluon plasma can, however, only survive if the energy per baryon of the strange-quark object is smaller than the energy per baryon of the corresponding hadronic system at the same $f_{s}$. For the bag model the strangelet is stable for $B^{1 / 4}=145 \mathrm{MeV}$ or metastable for $B^{1 / 4}=160-180 \mathrm{MeV}$ [9].

In the following we use $m_{s}=150 \mathrm{MeV}$ and $A\left(t_{0}\right)=100$ for the sake of illustration. Figure 4 gives an impression how the hadronization proceeds in the present model. The initial parameters are a net strangeness content of $f_{s}\left(t_{0}\right)=0.25$ and a moderate entropy per baryon ratio $(S / A)\left(t_{0}\right)=10$ :

A cold strangelet emerges from the expansion and evaporation process with an approximate baryon number of $A \sim 20$ (see also Fig. 5), a radius of $R \sim 2.5 \mathrm{fm}$, and a net strangeness fraction of $f_{S}(t \rightarrow " \infty$ ") $\sim 1.4$, i.e., a chargeto-baryon ratio $Z / A=\left(1-f_{s}\right) / 2 \sim-0.2$. This would be an interesting object also from its global properties: It would comprise a nucleus baryon number, but negative charge. It has recently been speculated [24] that similar objects could be formed in heavy-ion collisions also via multiple conventional coalescence of $\Sigma^{-}$hyperons with

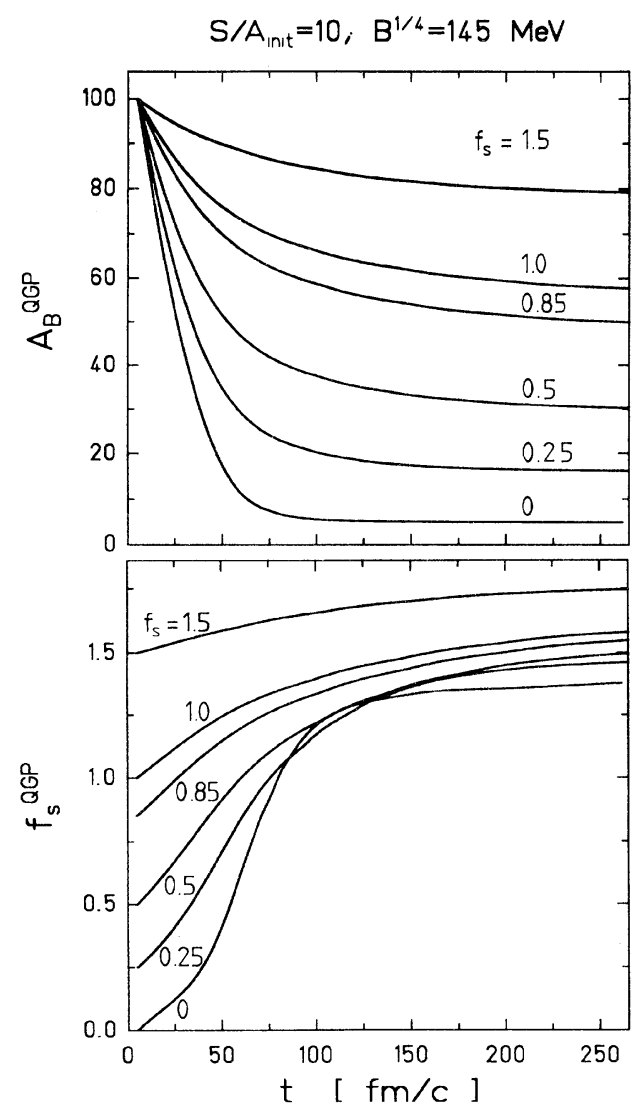

FIG. 5. Baryon number and strangeness content for the same parameters as in Fig. 4, but for different initial values of the net strangeness content $f_{s}\left(t_{0}\right)$. baryons into so-called metastable exotic multistrange objects (MEMO's).

Such an object, be it a strangelet consisting of deconfined quarks or a MEMO consisting of hyperons and nucleons, would be particularly easy to separate experimentally from the background: Because of its opposite charge, it could easily be separated magnetically from the whole (hyper)nuclear background with positive charge, and it would deposit much less energy (about $2 \mathrm{~A}$ $\mathrm{GeV}$ ) in a calorimeter than an antinucleus of roughly the same $A$ and velocity.

Temperature and entropy decrease drastically throughout the hadronization process (see Fig. 4). Thus the pressure goes to zero and an absolutely stable, cold strangelet is created. The energy per baryon of the remaining strange-quark droplet converges to its zerotemperature value.

The number of emitted particles is also plotted. There are only two kaons and three hyperons produced. This is due to the low initial entropy per baryon. No additional kaon evaporation is expected in this case, although these kaons are ernitted at the very beginning. The $s$ quarks stay in the quark-matter phase because the $\Lambda$ is too heavy to be emitted in large measures.

The initial net strangeness is varied in Fig. 5 to study the influence of $f_{s}\left(t_{0}\right)$ as compared to Fig. 4, where $f_{s}\left(t_{0}\right)=0.25$ has been assumed. It is important to point out that a small strangelet of $A_{B}^{\mathrm{QGP}}(t \rightarrow \infty) \sim 5$ with $f_{s} \sim 1.2$ survives even if $f_{s}\left(t_{0}\right)=0$. Although the initial entropy $S / A=10$ is small, $\sim 6$ kaons have been emitted conserving the total strangeness. This underlines the important role played by the kaons during hadronization. More kaons (and of course also pions) are produced and therefore also emitted when the initial entropy per baryon is higher.

This is demonstrated in Fig. 6(a) with $f_{s}^{\mathrm{QGP}}\left(t_{0}\right)=0$ where the initial entropy $S / A$ is varied: Observe that the higher the initial entropy, the larger the baryon content $A$ of the surviving strangelet. For $S / A=25$, a rather large strangelet of $A \sim 18$ is distilled. For very high initial entropies, $S / A=50$ and $S / A=100$, respectively, we find in addition that the baryon number of the surviving strangelet still increases to $A \sim 21$ and $A \sim 25$, respectively. Again negatively charged strangelets result for all cases presented. $f_{S}(t \rightarrow \infty) \sim 1.3-1.5 ; Z / A$ $\sim(-0.15)-(-0.25)$.

Note that for $S / A<7.5$, there is too little thermal excitation in the system for the distillation mechanism to work: It relies heavily on the emission of antistrangeness (via kaons) to separate $s$ from $\bar{s}$. If the temperature is insufficient for surface evaporation, this separation is not possible. Then the net strangeness ratio $f_{s}$ of the system does not increase, but stays close to zero. The very fact that a large plasma droplet survives in this case is due to the small $B^{1 / 4}$ value (145 MeV), which makes quark matter (consisting of $u$ and $d$ quarks only) energetically slightly more favorable than normal nuclear matter at zero temperature.

This behavior changes if we increase the bag constant only slightly to $150 \mathrm{MeV}$; strange-quark matter is still absolutely stable, nonstrange-quark matter is not. There- 
fore quark droplets are not distilled for small entropies $S / A<7.5$, when there is no initial net strangeness, i.e., $f_{s}\left(t_{0}\right)=0$.

This surprising prediction offers the opportunity to look for strangelets at much higher bombarding energies than expected naively $[8,9]$. A high initial entropy does not necessarily prohibit strangelet formation. This con- clusion is in contrast with the claim [25] that the distillation idea $[8,9]$ may never work due to the large entropy content in the QGP. Abundant kaon production enriches the plasma rapidly with net strangeness at high entropies. Pions are also necessary for a cooling of the remaining plasma blob.

In Fig. 6(b) the number of emitted particles is shown
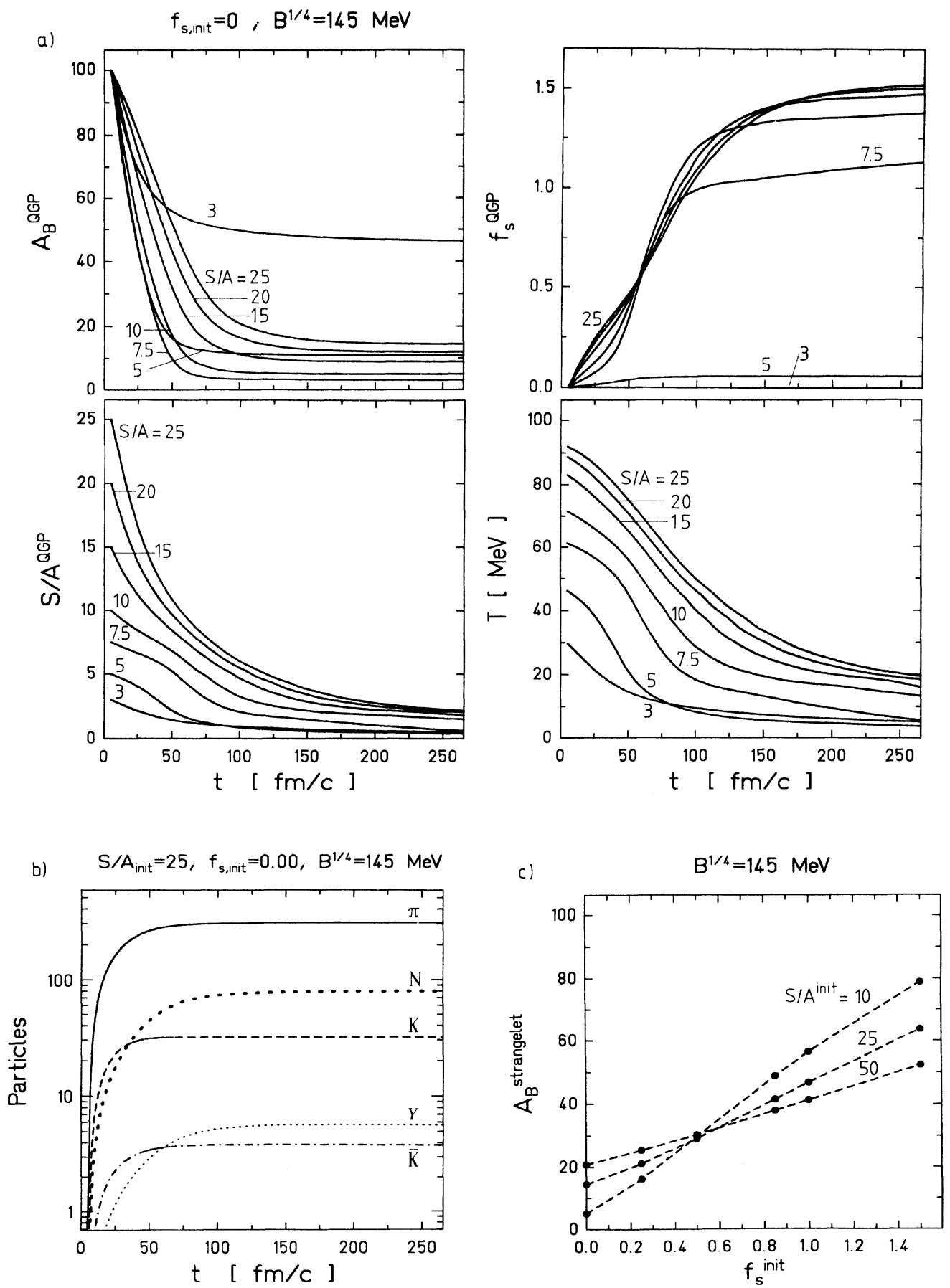

FIG. 6. (a) Strangelet distillation calculation for $B^{1 / 4}=145 \mathrm{MeV}, f_{s}\left(t_{0}\right)=0$ and several entropy ratios; (b) the produced particles for $(S / A)\left(t_{0}\right)=25$; and (c) the surviving mass number of the strangelet are depicted for the case of an absolutely stable strangelet (i.e., $B^{1 / 4}=145 \mathrm{MeV}$ ) for several initial configurations. 
for the special case $(S / A)\left(t_{0}\right)=25$, where a strangelet condenses after $\sim 200 \mathrm{fm} / c$ : Roughly 300 pions and 32 kaons are produced prior to most of the other hadron species. Only nine particles with positive strangeness $(\sim 3 \bar{K}, \sim 6 Y)$ are emitted, thus leaving some additional 23 units of strangeness in the strangelet. This surplus in strangeness is contained in the missing strangelet. The total $K^{+} / \pi^{+}$ratio is approximately 0.16 , in this situation. The pions carry about half of the available entropy off the system.

This high surplus in the kaon number would give $f_{s}^{\text {tot }}\left(t_{0}\right) \sim 0.25-0.3$ for $A \sim 100$, if a comparable number is emitted from the plasma before phase transition takes place. However, for these higher temperatures, an even larger surplus can be expected. The result for an initial strangeness fraction $f_{s}^{\text {tot }}\left(t_{0}\right) \sim 0.25$ can be read off Fig. 6(c). The baryon number of the strangelet has increased to $A_{B}^{\text {QGP }} \sim 20-30\left(f_{s} \sim 1.3\right)$, as compared to the results of Fig. 6(a).

In addition, the strangelet mass after a complete evolution is plotted versus various initial, ad hoc unknown parameters of the fireball, namely the initial entropy per baryon number and the net strangeness ratio of the plasma fireball due to the preequilibrium kaon evaporation out of the plasma before phase transition takes place. Observe once more that the higher the initial entropy, the larger the baryon content $A$ of the surviving strangelet. This is also true for the initial net strangeness fraction. Realistic parameters for the entropy may be larger than $\sim 10$ and for the net strangeness, as mentioned earlier, probably less or equal 0.5 . Hence, one might expect a reasonable strangelet with a baryon number $\sim 10-30$.

How does the situation change when the bag constant is increased to $B^{1 / 4}=160 \mathrm{MeV}$ and $B^{1 / 4}=180 \mathrm{MeV}$, i.e, for metastable strangelets? Figure 7 (a) analyzes the evolution for $f_{s}\left(t_{0}\right)=0.25$ and $B^{1 / 4}=160 \mathrm{MeV}$ for various initial entropy parameters. As expected, large strangelets may barely survive only for higher initial entropies $\left(A \sim 10, f_{s} \sim 1.8-2, Z / A \sim-0.45\right)$. However, larger strangelets turn out to be produced also for $S / A=3$ and 5 . This is due to the initial surplus of $25 s$ quarks assumed. For these small excitation energies additional kaons cannot be emitted, for the same reasons as discussed with respect to Fig. 4. In Fig. 7(b) the surviving mass number versus the initial parameters is plotted for this bag constant. If we assume that no initial evaporation from the hot QGP has occurred, i.e., as the phase equilibrium is reached there are as many $s$ as $\bar{s}$ in the plasma phase $\left[f_{s}^{\mathrm{QGP}}\left(t_{0}\right)=0\right]$, then strangelets with moderate initial entropy do not survive. There seems to be a chance only for the higher $S / A$ values.

Some reheating is observed in Fig. 7(a) for $S / A \leq 10$ at the beginning of the evolution [compare Fig. 2(a)]. Entropy conservation in fact reheats the system [16], in agreement with the findings of Barz et al. [13]. However, nucleon evaporation is no longer energetically favored beyond a critical point in the strangeness content $f_{s}$ if these smaller bag constants are used: This critical point corresponds to the so-called tangent point at $f_{s} \sim 0.7-0.9$ [9]. Additional nucleon evaporation must strictly cool the remaining system as demonstrated in the introduction because $S^{\mathrm{QGP}} / A<S^{\text {had }} / A$. This occurs only for the small bag constants less than $B^{1 / 4} \leq 190 \mathrm{MeV}$ and where the phase transition occurs at moderate temperatures $\lesssim 100 \mathrm{MeV}$. In the late stage of the evolution the temperature falls to $T \sim 20-30 \mathrm{MeV}$. Then, the specific hadron entropy $S^{\text {had }} / A$ can become quite large and the density of the hadron phase becomes small, $\rho \rightarrow 0$. The temperature drops like $T \sim \rho^{-2 / 3}$ in an isentropic expansion of an ideal gas [26]. On the other hand, the entropy in the plasma droplet, $S^{\mathrm{QGP}} / A$, approaches 0 for small temperatures because of its large density.

A calculation for $B^{1 / 4}=180 \mathrm{MeV}$ is shown in Fig. 7(c) for the sake of completeness. As discussed in the detailed investigation of zero-temperature strange-quark matter [9], the strangelet formation can work in principle only for bag constants $B^{1 / 4}<190 \mathrm{MeV}$; otherwise, the QGP always converts completely into hadrons, because of the unfavorable high mass of the strangelet. But with bag constants close to $190 \mathrm{MeV}, S / A$ has to be quite small (in our example $S / A<7.5$ ) to make strangelet distillation feasible. However, plasma formation at small entropies and sufficient kaon evaporation are probably hard to achieve simultaneously in ultrarelativistic heavy ion collisions. Therefore barely metastable strangelets are less favored in such experiments.

We would like to illuminate the physical origin of the dynamical "overshooting" of the final $f_{s}^{\text {strangelet values }}$ $\left(f_{s}^{\mathrm{QGP}}>1\right)$ predicted here: Even if the minimum of the energy per baryon of strange quark matter at finite temperatures versus the strangeness fraction $f_{s}$ is at values of $f_{s} \sim 0.8$, the total energy per baryon can still be lowered by separating more nucleons from the strange-matter droplet. Then the strangeness fraction in the remaining smaller droplet is enriched to $f_{s}^{\mathrm{QGP}} \gtrsim 1$. This argument is analogous to the tangent construction used in [9] for zero temperature. For finite temperatures the surplus in thermal energy causes additional nucleon evaporation, so that the tangent point will be "overshot." Therefore the strangelets can have a strangeness fraction exceeding 1 . The hyperons, on the other hand, are still too heavy to be copiously produced as compared to the nucleons, as long as the bag constant is less than $B^{1 / 4} \lesssim 180 \mathrm{MeV}$. For larger bag constants, i.e., $B^{1 / 4}>180 \mathrm{MeV}$, energy can be gained also by $\Lambda$ or $\Sigma$ emission and consequently, the quark-gluon plasma evaporates completely.

Figure 8 depicts the evolution in time of the chemical potentials, the final values for $\mu_{q}$ were taken as input for the estimate [Eq. (2)] given in the introduction. One observes that their values approach "rather" quickly their asymptotic numbers, even when the temperature of the strangelet is still not so small, $T \sim 40-60 \mathrm{MeV}$. The strange chemical potential turns out to be larger than the quark chemical potential in both cases (absolutely stable or metastable). This fact was just mentioned above. The evaporation of the particles forces these potentials to become quasistationary. So, Eq. (2) is a fairly good estimate for the final baryon number of the strangelet.

From Fig. 2(b) one is tempted to expect that the droplet may reach these values at smaller temperatures where $f_{s}^{\text {QGP }} \equiv f_{s}^{\text {had }}[14]$. However, the loss in entropy increases 
relatively to the increase in the strangeness content of the droplet, because $(S / A)^{\text {had }}$ gets larger and larger. The cooling process has stopped the emission of further nucleons. Also note that the final values of the potentials are only minor sensitive to the initial entropy per baryon content of the system. Therefore, the charge-to-mass ratio of the surviving strangelet is nearly constant.
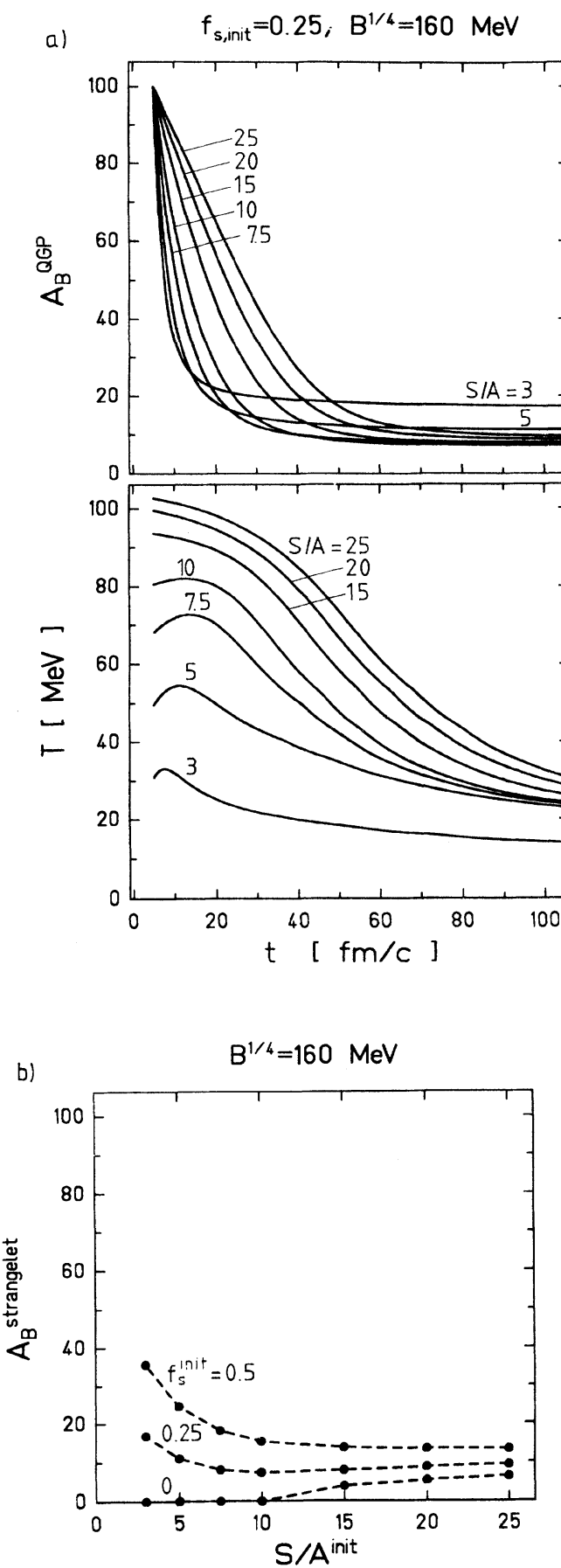

To summarize our model results: The strangelet distillation process from a QGP with initial net strangeness zero turns out to work only for bag parameters $\left(B^{1 / 4}<190 \mathrm{MeV}\right)$ which allow for stable or metastable strange-quark-matter droplets with nonzero strangeness at zero temperature. We emphasize the importance of kaon and pion evaporation. They favor the creation of a
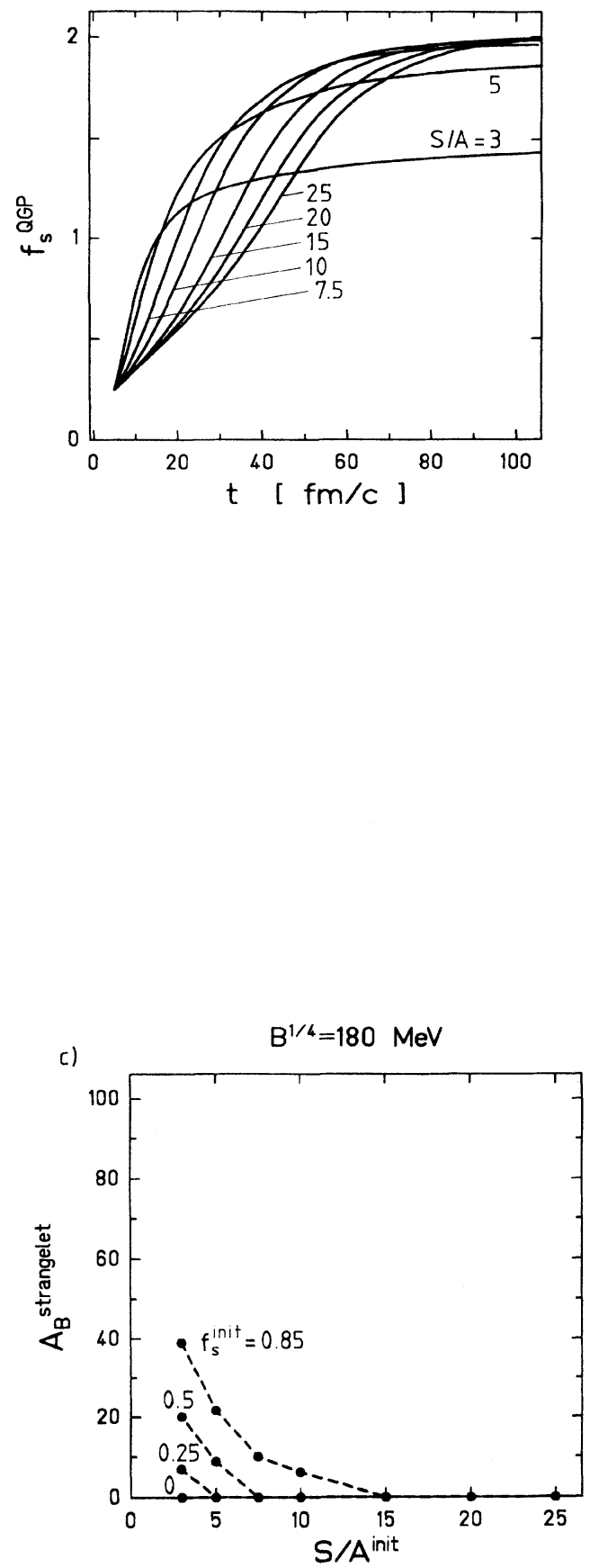

FIG. 7. (a) Strangelet distillation calculation for $B^{1 / 4}=160 \mathrm{MeV}$; (b) the surviving mass number of the strangelet for this metastable case; (c) as in (b), however, for the barely metastable case, i.e., $B^{1 / 4}=180 \mathrm{MeV}$. 


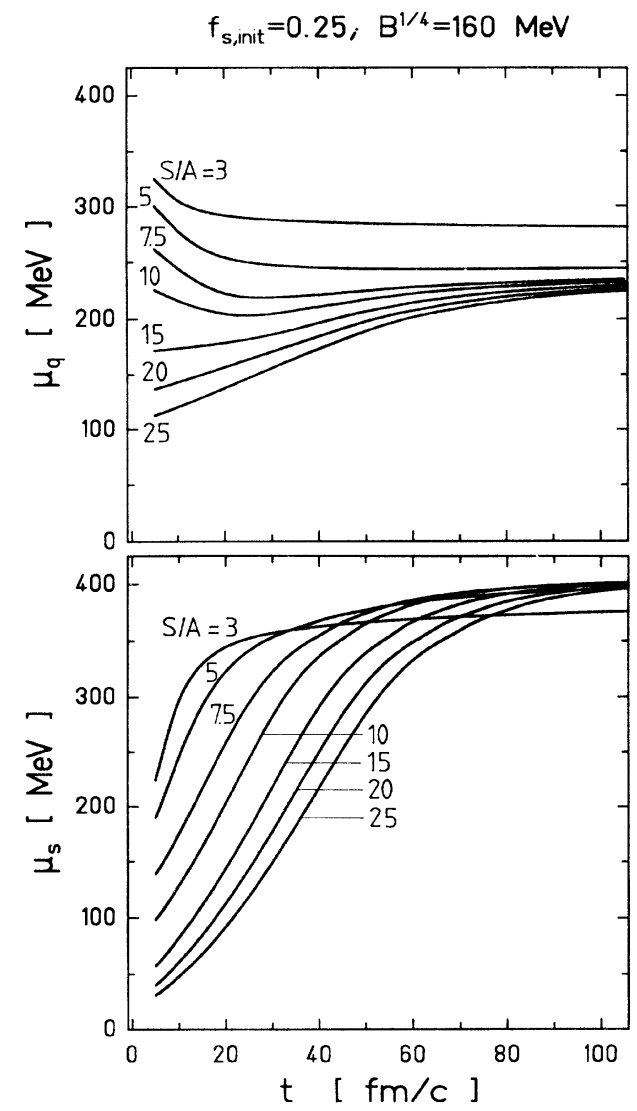

FIG. 8. The evolution in time of the chemical potentials $\mu_{q}, \mu_{s}$ is depicted for one of the presented calculations [see Fig. 7(a)].

strangelet and allow for the necessary cooling.

In principle the cooling due to the photons has to be included in the calculations. This would yield a faster cooling of the strangelets at large times. We have neglected this process for the sake of simplicity.

Two observations are remarkable: The distillery works even for larger initial entropies $S / A=50$ or 100 . This suggests that strangelets could also be candidates for observing the QGP at the future CERN and BNL Relativistic Heavy Ion Collider (RHIC) facilities with heavy systems. The second feature is the fact that the charge of the strangelet (with positive baryon number) can be negative. The baryon density of the strangelet would be about 1.5-2 of normal nuclear matter density.

\section{CONCLUSIONS AND OUTLOOK; WHERE TO LOOK FOR STRANGELETS?}

We conclude that there are two essential processes which favor the formation of strangelets from a baryonrich QGP formed in ultrarelativistic heavy-ion collisions. The first (as a prerequisite for strangelet production) is the $s-\bar{s}$-separation mechanism [8]. This process is not too sensitive to the bag constant used: It even works for $B^{1 / 4}=235 \mathrm{MeV}[8,13,21]$. This leaves us with a quark phase in the coexistence region of hadronic and quark matter, which is charged up with strangeness. The $s$ quarks, created in pairs with $\bar{s}$ quarks in the early quarkgluon fireball, remain in the quark phase during the phase transition, the $\bar{s}$-quarks materialize into kaons. The second is the evaporation of the hadronic gas with its (relative) antistrangeness excess, which charges the remaining system with net strangeness.

The observation that the distillation works even for higher entropies offers, contrary to our earlier remarks $[8,9]$, the opportunity to look for strangelet production at the highest bombarding energies available in the future for very heavy systems, e.g., at the CERN SPS $\left(E_{\text {lab }} \sim 200\right.$ $\mathrm{GeV} /$ nucleon) or at RHIC ( $E_{\text {lab }} \sim 20 \mathrm{TeV} /$ nucleon).

An important prediction is the negative charge-to-mass ratio, $Z / A \sim-0.1$ for absolutely stable strangelets, and $Z / A \sim-0.45$ for metastable droplets. If strangelets were actually produced in heavy-ion collisions, it would be the only unique signal for the formation of a QGP. It then could be detected by its unusual negative charge-tomass ratio $(-0.1<Z / A<-0.5)$. It can also be seen by interaction with a secondary nucleus: multiple production of $\Lambda$ 's $\Sigma$ 's, $\Xi$ 's, and $\bar{K}$ 's in such a secondary reaction would signal its existence.

Yet the question still remains, on which time scale weak decay or flavor-changing modes will appear. How would a strangelet then look like when passing through the detector? A magnetic spectrometer with dimension $\sim 10 \mathrm{~m}$ sets a natural time scale $\sim 10^{-7} \mathrm{sec}$. The weak decay modes of strangelets have been investigated by Chin and Kerman [11] and more elaborately, but only for the hypothetical six-quark state, the $H$ dibaryon, by Donoghue et al. [27]. In the following we adopt the reasoning of Ref. [11].

If the produced strangelet is absolutely stable $\left(f_{s} \sim 1.1-1.2, B^{1 / 4}=145 \mathrm{MeV}\right)$ the only energetically

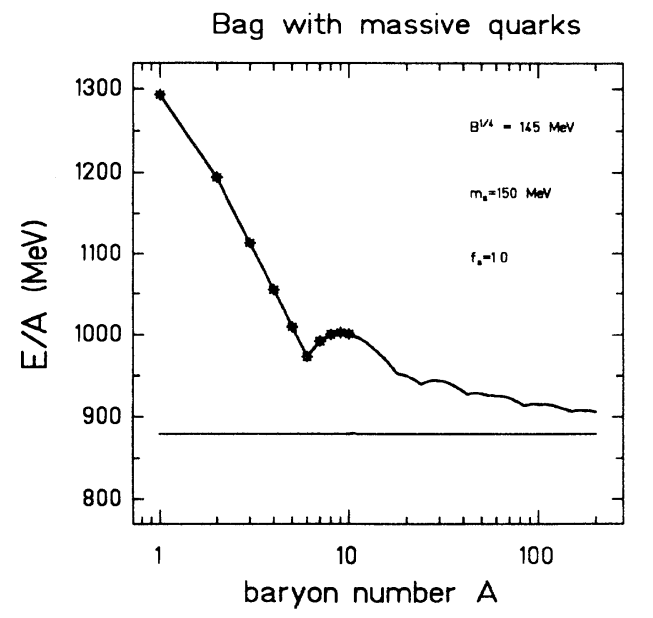

FIG. 9. $E / A$ vs $A$ in a hadronic (MIT) bag model for noninteracting quarks. A constant strangeness fraction $f_{s}=1$ is assumed $\left(m_{s}=150 \mathrm{MeV}, B^{1 / 4}=145 \mathrm{MeV}\right)$. 
possible decay mode is the weak leptonic decay $(s \rightarrow d$, $\left.Q \rightarrow Q^{\prime}+e+\bar{v}\right)$, which will turn the strangelet to its minimum value in energy at $f_{s} \sim 0.8$ (if the quarks are assumed to have no residual interaction [9]). Chin and Kerman estimated the time scale for this weak process to be $\sim 10^{-4}$ sec. Hence, the strangelet will remain in its initial condition.

The situation turns out to be a little bit more complicated, if the strangelet would be metastable $\left(f_{s} \sim 1.7-1.9\right.$, $\left.B^{1 / 4}=160 \mathrm{MeV}\right)$. The weak nucleon decay, $s \rightarrow d$, $Q \rightarrow Q^{\prime}+n$ is energetically possible in the range $0.5<f_{s}<1.7$. For $f_{s}>1$, it would enrich the quark droplet with strangeness. The strangelet will then also remain approximately at its initial conditions, $f_{s} \sim 1.7-1.9$, since the weak leptonic decay mode is too slow. Hence, metastable strangelets may be seen with $Z / A \sim-0.45$.

We want to point out that these estimates apply only to large systems. For $A \sim 5-25$ there is a strong shell structure of strange-quark matter. Figure 9 presents the solution of the Dirac equation for a bag with MIT boundary conditions with $B^{1 / 4}=145 \mathrm{MeV}$, a vanishing coupling constant $\alpha_{s}=0$, a strange-quark mass of $m_{s}=150 \mathrm{MeV}$, and a constant strangeness fraction of $f_{s}=1$ (for details, see Ref. [7,9]). The decay modes of strangelets trapped in such a shell stabilized state are strongly suppressed. Large fluctuations around these average predictions can be expected, in particular for the strangeness production per event and for the evaporation process.

If the negatively charged strangelet could be slowed down, it may easily attract (due to Coulomb interaction) and absorb at once protons or light nuclei. The energy gained in such reactions can be carried away by highenergy photons or pions, which may be detected. In response, the quark droplet will increase its size and move towards smaller $f_{s}$ down to the minimum value of $f_{s} \sim 0.8$. At $f_{s}<1$ the absorption will stop because of Coulomb repulsion.

Let us speculate at the end about the existence of very small "mini-strangelets" with a sufficiently small mass, for example, the $\boldsymbol{H}$-dibaryon proposed by Jaffe [10]. Assume that $m_{H} \sim 2 m_{N} \ll 2 m_{\Lambda}$. If such low-mass strangemultiquark states would exist, the survival of massive strangelets would give way to an abundant production of the $H$. Such light states have not been observed today.

\section{ACKNOWLEDGMENTS}

We are indebted to D. H. Rischke and J. Schaffner for a critical reading of the manuscript and to $A$. Schäfer and P. Koch for valuable suggestions concerning the estimate of the lifetime of metastable strangelets. Discussions with H. Gutbrod, B. Povh, K. Pretzl, J. Sandweiss, H. Crawford, S. Nagamiya, F. Rotondo, R. Maika, and E. Quercigh on the possibilities of observing strangelets in heavy-ion collisions are gratefully acknowledged.
[1] Proceedings of Quark Matter '90 [Nucl. Phys. A (to be published)], and previous quark matter proceedings; The Nuclear Equation of State, Proceedings of the NATO Advanced Study Institute, edited by $W$. Greiner and $\mathbf{H}$. Stöcker, NATO ASI Series B: Physics, Vol. 216 A, B (Plenum, New York, 1989); for first experimental results on the search for strangelets created in relativistic heavy-ion collisions, see S. V. Greene and the E814 Collaboration, ibid. Vol. 216 B, p. 117.

[2] P. Koch, B. Müller, and J. Rafelski, Phys. Rep. 142, 167 (1986).

[3] T. Matsui and H. Satz, Phys. Lett. B 178, 416 (1986).

[4] NA38 Collaboration, Phys. Lett. B 251, 465 (1990).

[5] E802 Collaboration, in Proceedings of the Heavy Ion Physics at the AGS Workshop, Upton, New York, 1990.

[6] E. Witten, Phys. Rev. D 30, 272 (1984); A. R. Bodmer, ibid. 4, 1601 (1971).

[7] E. Farhi and R. L. Jaffe, Phys. Rev. D 30, 2379 (1984).

[8] C. Greiner, P. Koch, and H. Stöcker, Phys. Rev. Lett. 58, 1825 (1987).

[9] C. Greiner, D. H. Rischke, H. Stöcker, and P. Koch, Phys. Rev. D 38, 2797 (1988).

[10] R. L. Jaffe, Phys. Rev. Lett. 38, 195 (1977).

[11] S. A. Chin and A. K. Kerman, Phys. Rev. Lett. 43, 1292 (1979).

[12] H. Liu and G. L. Shaw, Phys. Rev. D 30, 1137 (1984).
[13] H. W. Barz, B. L. Friman, J. Knoll, and H. Schulz, Phys. Lett. B 242, 328 (1990).

[14] J. Madsen, H. Heiselberg, and K. Riisager, Phys. Rev. D 34, 2947 (1986).

[15] H. W. Barz, B. L. Friman, J. Knoll, and H. Schulz, Nucl. Phys. A484, 661 (1988).

[16] P. R. Subramanian, H. Stöcker, and W. Greiner, Phys. Lett. 137B, 468 (1986); U. Heinz, P. R. Subramanian, H. Stöcker, and W. Greiner, J. Phys. G 12, 1237, (1986).

[17] J. Kapusta and A. Mekjian, Phys. Rev. D 33, 1304 (1986).

[18] G. Bertsch, M. Gong, L. D. McLerran, V. Ruuskanen, and E. Sarkkinen, Phys. Rev. D 37, 1202 (1988).

[19] R. Hagedorn and J. Rafelski, Phys. Lett. 97B, 180 (1980).

[20] J. D. Bjorken, Phys. Rev. D 27, 140 (1983).

[21] J. Rafelski, Phys. Lett. B 190, 167 (1987).

[22] B. L. Friman (unpublished).

[23] B. Müller and J. M. Eisenberg, Nucl. Phys. A435, 791 (1985).

[24] J. Schaffner, C. Greiner, and H. Stöcker (in preparation).

[25] U. Heinz, K. S. Lee, and M. J. Rhoades-Brown, Mod. Phys. Lett. A 2, 153 (1987).

[26] L. D. Landau and E. M. Lifshitz, Lehrbuch der Theoretischen Physik 5, Statistische Physik (Akademie-Verlag, Berlin, 1979).

[27] J. F. Donoghue, E. Golowich, and B. R. Holstein, Phys. Rev. D 34, 3434 (1986). 